\title{
Immunohistochemical Evaluation of Angiogenesis Related Markers in Pyogenic Granuloma of Gingiva
}

\author{
MaryamSeyedmajidi', Shahryar Shafaee ${ }^{2}$, Golnarsadat Hashemipour ${ }^{3 *}$, Ali \\ Bijani $^{4}$, Hodis Ehsani ${ }^{5}$
}

\begin{abstract}
Background: Pyogenic granuloma is a common non-neoplastic connective tissue proliferation. ICAM-1 and VCAM-1 are vascular adhesion molecules and CD34 is a marker for evaluation of angiogenesis. The purpose of this study was to compare the immunohistochemical expression of ICAM-1, VCAM-1 \& CD34 in oral pyogenic granuloma and normal gingiva. Materials and Methods: This study was performed on thirty five formalin-fixed, paraffin embedded samples of gingival pyogenic granuloma. Also we used thirty five paraffined blocks of normal gingiva as control group which were taken from crown lengthening surgery. We employed immunohistochemistry staining for our prepared microscopic slides using monoclonal mouse anti-human antibodies against ICAM-1 (CD54), VCAM-1 (CD106) and CD34. Slides were examined under light microscope and then the mean amount of stained vessels also known as microvascular density (MVD) in highly vascularized areas (hot spots) was measured. Paired $t$-test and repeated measures ANOVA were used to compare the difference between quantitative variables and Chi-square test for qualitative variables in different groups. Pearson correlation coefficient was used to compare relations between quantitative variables. $P<0.05$ was considered significant. $\underline{\text { Results: }}$ The mean of MVD for ICAM-1, VCAM-1 and CD34 was significantly higher in pyogenic granuloma than normal gingiva $(p<0.001 \& p<0.001 \& p<0.001$, respectively). Expression of $\mathrm{CD34}$ in pyogenic granuloma was significantly higher than ICAM-1 and VCAM-1 (P<0.001). Besides, expression of ICAM-1 in normal gingiva, was significantly lower than two other markers $(\mathbf{p}<\mathbf{0 . 0 0 1 )}$. Conclusions: Regarding the results, it seems that ICAM-1, VCAM-1 and CD34 are useful biomarkers in evaluation of vascular and inflammatory lesions such as gingival pyogenic granuloma and the results indicate the role of these biomarkers in pathogenesis of oral pyogenic granuloma.
\end{abstract}

Keywords: Pyogenic granuloma - angiogenesis - microvascular density - ICAM-1 - VCAM-1 -CD34

Asian Pac J Cancer Prev, 16 (17), 7513-7516

\section{Introduction}

Pyogenic granuloma (PG) is a common kind of tumorlike lesion of oral cavity that caused by local irritation or trauma Neville (2009). It is a non-neoplastic process. Owing to the existence of hyperplastic granulation tissues which contain lots of capillaries, it appears as a red mass (Vassilopoulos et al., 2011). Pyogenic granuloma is more prevalent in children and young adults. It has a tendency to occur in Gum (Nelson and Thompson, 2007). Most studies have shown that this lesion occurs predominantly in women. It is probably due to influences of female hormones on blood vessels Kawachi (2011). Hormonal changes during puberty and pregnancy possibly transform the gingival reparative response to injury, leading to growth of a lesion in pregnancy period, which is called Granuloma Gravidarum or gestational tumor (Regezi and Sciubba, 2012). The etiology of pyogenic granuloma can be attributed to an imbalance between reinforcing factors and inhibitors of angiogenesis (Epivatianos et al., 2005).

Adhesive vascular factors are proteins which allow to leukocytes to adhere to the vascular wall. Intercellular adhesion molecule-1 (ICAM-1) or CD54 is a glycoprotein of the immunoglobulin family that stimulates immunologic and inflammatory reactions (Perschbacher and JacksonBoeters, 2004). ICAM-1 expression is increased in inflammatory conditions by specific mediators. Leukocyte function antigen-1 (LFA-1) is a recipient of ICAM-1 and a member of the integrins family that plays a significant role in adherence of leukocytes to vessel walls. Connection between ICAM-1 and LFA1 stimulates several reactions including leukocyte response to antigen, connection of leukocytes to endothelium and their immigration (AL-Rawi and Younis, 2007). Although cytokines with inductor effects increase expression of ICAM-1 during inflammatory conditions, endothelial cells, leukocytes and

${ }^{1}$ Dental Materials Research Center, Dental Faculty, ${ }^{2}$ Cellular \& Molecular Biology Research Center, ${ }^{3}$ Oral \& Maxillofacial Pathology Department, Dental Faculty, ${ }^{4}$ Non-communicable Pediatrics Diseases Research Center, Babol University of Medical Sciences, Babol, ${ }^{5}$ Periodontology Department, Dental Faculty, Mazandaran University of Medical Sciences, Sari, Iran * For correspondence: hashemipoorgolnar2@gmail.com 
lymphocytes express a small amount of ICAM-1 in normal conditions (Perschbacher and Jackson Boeters, 2004).

Vascular cell adhesion molecule-1 (VCAM-1) or CD106 is One of the most important mediators of vascular adherence that controls immune responses. Very late antigen-4 (VLA-4) is a VCAM-1 recipient. VCAM-1 expresses at the surface of mononuclear cells and its expression in endothelial cells shows the increase of cell adhesion and migration of active mononuclear cells (Al-Rawi and Al-Kawas, 2010). VCAM-1 and Selectin mediate initial adherence of leukocytes and their migration from blood vessels. This action gets strengthened by ICAM-1. Although ICAM- 1 is induced by proinflammatory cytokines such as IL- $1 \beta, \mathrm{TNF}-\alpha, \mathrm{IFN}-\gamma$, IL-2 and E-coli lipopolysaccharides , VCAM-1 is induced just by IL-1ß (Xue et al., 2005).

CD34 is a membranus glycoprotein that is expressed at the surface of stem cells and hematopoetic cells of bone marrow and endothelium in non-hematopoetic organs and embryonic fibroblasts (Ancuta et al., 2010). Its main function is increasing intercellular connection (Vasconcelos et al., 2011). It is used for evaluation of vascularization in normal and neoplastic tissues (Lavu and Padmavathy, 2009).

Angiogenesis is described as formation of new blood vessels that is controlled through a series of stimuli and inhibitors and it cooperates in establishing of homeostasis (Saghafi et al., 2011). Many studies have indicated the role and importance of angiogenesis in chronic inflammatory diseases. It enhances vessel budding, oxygen delivery and intensifies turnover of the cells involved in inflammation (Kademani et al., 2009). Tumor angiogenesis can be measured by Microvascular density (MVD). Number of vessels are counted in the regions of the lesion that contain high number of vessels (hot spots) (Rykala et al., 2011; Ullah et al., 2013).

ICAM-1 and VCAM-1 are increased in inflammatory processes and CD34 is a factor for evaluation of vascularity (Ancuta et al., 2010). All of these factor should be measured by immunohistochemical assay (Palit and Inamadar, 2011). Therefore, the present study was carried out to evaluate angiogenesis in pyogenic granuloma as an inflammatory lesion by using immunohistochemical assay.jjiimj

\section{Materials and Methods}

This cross-sectional descriptive and analytical study was implemented on thirty five formalin-fixed, paraffin embedded samples of gingival pyogenic granuloma and thirty five formalin-fixed, paraffin embedded samples of normal gingiva that taken from Crown Lengthening Surgery considered as the control group which achieved from the archives of oral and maxillofacial pathology department of Babol dental faculty during 2004-2014. Samples of pyogenic granuloma that related to pregnant patients, patients with systemic disorders and also fibrotic(healed) pyogenic granulomas were excluded.

Paraffined blocks of pyogenic granuloma after providing microscopic slides, stained with H\&E to confirm the diagnosis and sufficiency of the sample volume.
Sections with $4 \mu \mathrm{m}$ thickness from paraffined blocks were prepared for the immunohistochemical study. The sections were deparaffinized and put in microwave in citrate buffer $(\mathrm{PH}=6.1)$ for 15 minutes to antigen retrieval. Then, they were washed in Tris Buffered Saline (TBS) $(\mathrm{Ph}=7.2)$ and treated with $3 \%$ hydrogen peroxide for 10 min to block the endogenous peroxidase activity. After, the sections were incubated with diluted primary antibodies for 1 hour followed by TBS wash, using diluted (1:50) monoclonal mouse anti-human ICAM-1 (CD54)(MEM111, ab2213, Abcam, UK)\& monoclonal mouse antihumanVCAM-1 (CD106) (EPR5047, ab134047, Abcam, UK)\& monoclonal mouse anti-human CD34 (12934349, Serotec,UK).Secondary antibodies (Rabbit anti mouse Immunoglobulin (IgG), abcam,UK) were applied and incubated for 30 minutes with substrated diaminobenzidine (DAB) as chromogen (liquid DAB+substrate chromogen system,Dako, Denmark). The sections were eventually washed and counterstained with Meyer's Hemotoxylin.

Negative control was gained with omission of primary antibodies and tonsillar tissue was considered as positive control. In order to evaluation of biomarkers staining, counting of positive cells was done by two independent pathologists and the mean of their results, was carried to account.

The number of vessels was counted according to Weidener's method and its further modifications (Foote et al., 2005). At first, slides were estimated under light microscope (Olympus BX41, Tokyo, Japan) at 100× magnification to achieve 5 areas with the largest number of vessels (hot spots). Then, number of vessels that stained with ICAM-1,VCAM-1 and CD34 was counted at 400× magnification. Then the average value of the vessel count (mean $\pm \mathrm{SD}$ ) in five fields for each case was determined and used as the final MVD value (microvascular density).

For counting the number of vessels stained with CD34, any single endothelial cell or group of endothelial cells which positively coloured, were evaluated as positive results of immunohistochemistry. Even in the cases that were without lumen formation, if just one stromal cell was found between endothelial cells, it decleared as positive. Vessel branches and non-continuous structures of the vessels also were considered as positive during our counting.

The differences of MVD between pyogenic granuloma and normal gingiva were measured and statistically analyzed (Paschoal et al., 2014). Data were analyzed by using SPSS17 statistical software. Paired t-test and Repeated Measures ANOVA were used to compare the difference between quantitative variables and Chi-square test for qualitative variables in different groups. Pearson correlation coefficient was used to compare relations between quantitative variables. $\mathrm{P}<0.05$ was declared significant.

\section{Results}

In the present study, paraffined blocks of thirty five patients with pyogenic granuloma of gingiva and also thirty five samples of normal gingiva were evaluated and demographic criteria of patients were presented in Table 1. 
The results of immunohistochemical staining for ICAM-1,VCAM-1 and CD34 expression in pyogenic granuloma and normal gingiva were examined and are presented in Table 2 (Figure1A-F).

ICAM-1,VCAM-1 and CD34 expression has been significantly more in pyogenic granuloma than normal gingiva $(\mathrm{p}<0.001 \& \mathrm{p}<0.001 \& \mathrm{p}<0.001$, respectively) (Figure 2).

Pearson correlation coefficient was measured between different variables. There was no significant correlation between age and gender of patients with expression of biomarkers. There were significant positive correlation between ICAM-1 and VCAM-1 expression ( $r=0.384$, $\mathrm{p}=0.023$ ) and also between VCAM- 1 and CD34 ( $\mathrm{r}=0.651$, $\mathrm{p}=0.000)$ in pyogenic granuloma. But correlation between ICAM-1 and CD34 was not significant $(\mathrm{r}=0.316, \mathrm{p}=0.064)$ (Figure 3).

\section{Discussion}

We found that, ICAM1, VCAM1 and CD34 expression was significantly higher in pyogenic granuloma in comparison with normal gingiva. In this study, adhesion molecules ofICAM-1 and VCAM-1 and also CD34 molecule as vascular indicators were measured to evaluate angiogenesis in pyogenic granuloma. Pyogenic granulomas represented increased expression of ICAM1,VCAM1 and CD34.

Regarding the fact that these biomarkers are expressed by endothelial cells and leukocytes, the increase of their expression can be attributed to the increase in the number of blood vessels and leukocytes in pyogenic granuloma (Seyedmajidi et al., 2013). Therefore, it can be concluded that angiogenesis plays a major role in ethiopathogenesis of pyogenic granuloma. Beginning of angiogenic responses as physiologic or pathologic processes is accomplished by activation of endothelial cells and cytokine release (Altamirano and Drut, 2008).

ICAM-1 is a member of immunoglobulin family which connects to LFA-1. This receptor is expressed by almost all of leukocytes in the blood circulation. Interaction between ICAM-1 and LFA-1 has an important role in adhesion and immigration of leukocytes (AL-Rawi and Younis, 2007).

VCAM-1 is also a member of immunoglobulin family. Its receptor is VLA-4 that is expressed on memory T-cells (Al-Rawi and Al-Kawas, 2010).

Weidner and his colleagues calculated MVD in regions with high concentration of blood vessels (hot spots) in breast cancer and this method has been done so far for other tumors (Foote et al., 2005). MVD is considered to be a suitable independent prognostic factor in cancerous patients. Evaluating expressions of angiogenic related markers may be an important step to study new therapies based on the use of anti-angiogenesis drugs in cancer treatment (Chien et al., 2006).

Al Rawi et al. in an immunohistochemical study measured expression of ICAM-1 and VCAM- 1 in pyogenic granuloma and indicated that these biomarkers have an effective role in angiogenesis and inflammation. From his point of view, the expression of VCAM-1 from endothelial cells is induced by TNF- $\alpha$ and IL- 1 in active inflammation and this expression play an important role in regulation of leukocytes immigration from blood circulation to lesion sites. TNF- $\alpha$ is produced by inductive macrophages via lipopolysacarides released from gram negative bacteria (Al-Rawi and Al-Kawas, 2010).

Scardina et al.(2009) also demonstrated the angiogenesis in oral lichen planus by immunohistochemical study using VCAM1, ICAM1,VEGF and CD34. Expression of these biomarkers in normal gingiva was much less, this matter could reflect the role of these molecules in the process of angiogenesis and inflammation (Scardina et al., 2009).

Lavu et al.(2009) in an immunohistochemical study on CD34 in normal gingiva indicated that CD34 is produced by vascular and epithelial stem cells in perivascular area and basal lamina of epithelium, also in our study perivascular endothelial cells in normal gingiva expressed CD34 (Lavu and Padmavathy, 2009).

Seyedmajidi et al. (2013) in a similar study that compared expression of ICAM-1 and VCAM-1 in oral lichen planus in comparison with normal mucosa, demonstrated higher level of these biomarkers in lichen planus as an inflammatory lesion wich is in accordance with our findings (Seyedmajidi et al., 2013).

In our study, higher expression of these biomarkers in pyogenic granuloma as a vessel-rich lesion compared with normal gingiva, illustrates the role of these molecules as angiogenesis related markers. Evaluation of immunohistochemical expression of angiogenesis related markers may be an important step for the study of new therapies based on the use of anti-angiogenesis drugs that are probably useful in diagnosis and treatment of vascular and inflammatory disease.

According to our study, higher expression of ICAM1,VCAM-1 and CD34 were seen in gingival pyogenic granuloma compared with normal gingiva. This higher expression appears to be involved in pathogenesis of oral pyogenic granuloma.

\section{Acknowledgements}

The present study is the result of a research project No. 9032034, approved by the Research Council of Babol University of Medical Sciences, and the thesis of a postgraduate student - Dr.Golnarsadat Hashemipour. The authors would like to thank the Deputy of Research and Technology of Babol University of Medical Sciences for financially supporting the project and also $\mathrm{Mr}$. Mohsen Aghajanpour in the Cellular and Molecular Biology Research Center of this university for his sincere cooperation in performing the immunohistochemical staining.

\section{References}

Ancuta C,Ancuta E,Zugun-Eloae F, et al (2010). Neoangiogenesis in cervical cancer: focus on CD34 assessment. Romanian J Morphol Embryol, 51, 289-94.

Al-Rawi N,Al-Kawas S, Younis W (2010). Immunolocalization of the cell adhesion molecules ICAM-1 \& VCAM-1 in pyogenic granuloma. Adv Med Dental Sci, 3, 92-7.

Altamirano E, Drut R (2008). Pyogenic granuloma of the 
Maryam Seyedmajidi et al transverse colon. Report of a pediatric case. Patologia, 46, 263-5.

AL-Rawi NH, Younis WH (2007). Immunohistochemical localization of intercellular cell adhesion molecule (ICAM-1) \& vascular cell adhesion molecule (VCAM-1) in Radicular cyst. Acad Sci J, MDJ, 4, 6-12.

Chien CY, Su CY, Hwang CF, et al (2006). High expressions of CD105 and VEGF in early oral cancer predict potential cervical metastasis. J Surg Oncol, 94, 413-7.

Epivatianos A, Antoniades D, Zaraboukas T, et al (2005). Pyogenic granuloma of the oral cavity: comparative study of its clinicopathological and immunohistochemical features. Pathology international, 55, 391-7.

Foote RL, Weidner N, Harris J, et al (2005). Evaluation of tumor angiogenesis measured with microvessel density (MVD) as a prognostic indicator in nasopharyngeal carcinoma: results of RTOG 9505. Int J Radiat Oncol Biol Physics, 61, 745-53.

Kawachi N (2011). A comparative histopathological and immunohistochemically study of capillary hemangioma, pyogenic granuloma and cavernous hemangioma in the oral region: with special reference to vascular proliferation factors. Int J Oral Med Sci, 9, 241-51.

Kademani D, Lewis JT, Lamb DH, et al (2009). Angiogenesis and CD34 expression as a predictor of recurrence in oral squamous cell carcinoma. J Oral Maxill Surg, 67, 1800-5.

Lavu V, Padmavathy R, Rao SR (2009). Immunolocalization of CD 34 positive progenitor cells in healthy human gingiva-a pilot study. Indian J Med Res (IJMR), 129, 685-9.

Neville BW (2009). Oral and maxillofacial pathology, Elsevier Brasil, 517-9.

Nelson BL, Thompson LD (2007). Sinonasal tract angiosarcoma: a clinicopathologic and immunophenotypic study of 10 cases with a review of the literature. Head Neck Pathol, 1, 1-12.

Paschoal JP,Bernardo V,Canedo NHS, et al (2014). Microvascular density of regenerative nodule to small hepatocellular carcinoma by automated analysis using CD105 and CD34 immunoexpression. BMC cancer, 14, 72 .

Palit A, Inamadar AC (2011). Immunohistochemistry: Relevance in dermatology. Indian J Dermatol, 56, 629.

Perschbacher K, Jackson Boeters L, Daley T (2004). The adhesion molecules NCAM, HCAM, PECAM-1 and ICAM-1 in normal salivary gland tissues and salivary gland malignancies. J Oral Pathol Med, 33, 230-6.

Regezi JA, Sciubba JJ, Jordan RC (2012). Oral pathology: clinical pathologic correlations. Elsevier Health Sci, 118-20.

Rykala J, Przybylowska K, Majsterek I, et al (2011). Angiogenesis markers quantification in breast cancer and their correlation with clinicopathological prognostic variables. Pathol Oncol Res, 17, 809-17.

Seyedmajidi M, Shafaee S, Bijani A, et al (2013). VCAM1 and ICAM1 expression in oral lichen planus. Int J Molecular Cellular Med, 2, 34 .

Saghafi S, Amoueian S, Montazer M, et al (2011). Assessment of VEGF, CD-31 and Ki-67 immunohistochemical markers in oral pyogenic granuloma: a comparison with hemangioma and inflammatory gingivitis. Iranian J Basic Med Sci, 14, 185-9.

Scardina G-A, Ruggieri A, Messina P, et al (2009). Angiogenesis of oral lichen planus: a possible pathogenetic mechanism. Med Oral Patol Oral Cir Bucal, 14, 558-62.

Ullah E, Nagi AH, Ashraf M (2013). Angiogenesis and mast cell density as predictors of patient survival in squamous cell carcinoma of lung. J Cancer Res Ther, 9, 701.

Vassilopoulos SI, Tosios KI, Panis VG, et al (2011). Endothelial cells of oral pyogenic granulomas express eNOS and CD105/ endoglin: an immunohistochemical study. J Oral Pathol Med, 40, 345-51.
Vasconcelos MG, Alves PM, Vasconcelos RG, et al (2011). Expression of CD34 and CD105 as markers for angiogenesis in oral vascular malformations and pyogenic granulomas. European Arch Oto-Rhino-Laryngol, 268, 1213-7.

Xue F, Zhang Y, Liu F, et al (2005). Expression of IgSF in salivary adenoid cystic carcinoma and its relationship with invasion and metastasis. J Oral Pathol Med, 34, 295-7. 